\title{
El Devenir Reciente de la Agricultura Familiar en el Capitalismo Agrario de la Región Pampeana Argentina
}

Melina Neiman'
Gabriel Bober

\section{Resumen}

El desarrollo del capitalismo agrario en la región pampeana involucró, de forma temprana, a pequeñas y medianas explotaciones agropecuarias de origen familiar, inmersas en la producción de commodities destinada a los mercados internacionales. Sin embargo, presentaban características que las distinguían de los grandes productores de la región, principalmente, por el origen familiar de su mano de obra. En su devenir histórico, estas unidades vieron transformadas tanto su organización laboral-productiva como sus dinámicas familiares. En este artículo se muestran estos cambios y se reflexiona acerca de la dificultad para definir a este actor social en función de sus antiguas particularidades pero, aún así, entender qué características lo siguen distinguiendo actualmente. El abordaje metodológico es cualitativo y se basa en el análisis de entrevistas en profundidad realizadas a productores, a sus hijos/as y esposas y a profesionales en el partido de Junín, provincia de Buenos Aires. Además se recurre a fuentes secundarias para explicar los factores relacionados con la expansión productiva y el precio de la tierra.

Palabras claves: Producción familiar. Región pampeana. Agricultura. Familias

\section{Introducción}

El desarrollo del capitalismo agrario en la región pampeana involucró, de forma temprana, a pequeńas y medianas explotaciones agropecuarias de origen familiar, inmersas en la producción destinada a los mercados internacionales de mercancías agropecuarias.

En su recorrido histórico, estas explotaciones familiares atravesaron diferentes etapas y presentaron características propias que las diferenciaron de

I Centro de Estudios e Investigaciones Laborales (CEIL) - Consejo Nacional de Investigaciones Científicas y Técnicas (CONICET) - Universidad de Buenos Aires (UBA). E-mail: mneiman@ceil-conicet.gov.ar

2 Centro de Estudios e Investigaciones Laborales (CEIL) - Consejo Nacional de Investigaciones Científicas y Técnicas (CONICET) - Universidad de Buenos Aires (UBA). E-mail: gbober@ceil-conicet.gov.ar 
otros sujetos sociales agrarios de la región, ya sea por las formas de tenencia de sus tierras, el uso del suelo, la dotación de capital o la conformación de la mano de obra.

En la primera parte de este artículo se presentan los contextos históricos y las principales características de las unidades productivas de "chacareros"3 desde que eran arrendatarios a fines del siglo XIX, hasta su posterior transformación en pequeńos propietarios a mediados del siglo XX.

Posteriormente se analizan, a través de información censal y de datos de coyuntura, los procesos socio-económicos desde los años '90 del siglo pasado y el impacto que tuvieron en estas unidades así como sus transformaciones en la organización laboral-productiva y en las dinámicas familiares.

Finalmente, se examina la forma en que se organiza la mano de obra de la explotación agropecuaria, las transformaciones en las modalidades de herencia y las condiciones que presentan las estrategias pluriactivas que desarrollan algunos miembros de las familias.

En este sentido, se busca dar cuenta de los cambios atravesados por estas unidades y reflexionar acerca de las dificultades para definir a este sujeto social en función de las antiguas particularidades pero también para entender las características que las siguen distinguiendo en la actualidad.

El abordaje metodológico es principalmente cualitativo y se basa en la realización y el análisis de entrevistas en profundidad. Se llevaron a cabo 30 entrevistas a titulares de explotaciones familiares, hijos/as y esposas de productores y profesionales del partido de Junín, ubicado en el noroeste de la provincia de Buenos Aires con una importante especialización agrícola orientada recientemente al cultivo de soja. Se recurre además a fuentes secundarias para explicar los factores relacionados con las transformaciones económicas, productivas y poblacionales. Entre estas fuentes se cuenta con los Censos Nacionales Agropecuarios de 1988 y 2002, diferentes informes privados de evolución económica y una encuesta a productores agropecuarios del partido de Junín realizada en el período 2008-20094.

3 Este actor fue denominado históricamente como "chacarero" debido a que arrendaba sus chacras en tierras de grandes propietarios.

4 Esta encuesta fue realizada en el marco de la tesis doctoral "La agricultura familiar en la región pampeana. Nuevos vínculos familiares y transformaciones en las unidades de producción". El corte por superficie de unidades de hasta 500 hectáreas equivalente al 90\% del total de unidades del partido, permite captar la mayor 


\section{El recorrido histórico de la agricultura familiar pampeana}

Hacia finales del siglo XIX y principios del XX, la imagen del agro pampeano era la de la convivencia de las grandes estancias ganaderas con los "chacareros" agricultores. En los estudios historiográficos, la llamada "visión tradicional" enfatiza la relación de subordinación de la pequeńa agricultura respecto de los terratenientes ganaderos, sintetizada en la imagen de una realidad agraria modernizada frente a otra atrasada que obstruía el desarrollo del capitalismo agropecuario: se contraponía la propiedad de la tierra con los chacareros arrendatarios y se consideraba a la región como una realidad homogénea, con procesos económicos-sociales comunes o únicos (Nemirowsky, 1933; Tenembaum, 1946; Oddone, 1956; Scobie, 1968). Además, quienes trabajaban la tierra eran, en general, los pequeños productores que organizaban su producción utilizando la fuerza de trabajo propia y la de su familia y que, a pesar de esfuerzos y privaciones, no lograban acumular capital para lograr un ascenso social (Balsa, 2006).

Sin embargo, los trabajos de Barsky y Pucciarelli (1991), Sartelli (1994), Zeberio (1993) y Balsa (1994) mostraron que, comenzando el siglo XX, el sector de los agricultores pampeanos no era tan homogéneo como señalaba la visión tradicional, que no toda la agricultura estaba subordinada a la ganadería, que había agricultores que contrataban mano de obra asalariada, que existía un segmento de unidades medianas que explicaban gran parte de la producción agrícola y que habían experiencias de acumulación. Estos "chacareros" ya eran sujetos capitalistas.

Sin duda, las primeras décadas del siglo XX fueron el período fundacional y de mayor intensidad del desarrollo agrícola pampeano. Para el 1881, menos del $2 \%$ de la zona norte de la provincia de Buenos Aires estaba sembrada con cultivos y la agricultura era inexistente en el oeste y sur de la provincia mientras que para 1937 ya se cultivaba el 60\% de la superficie de la zona norte, el $48 \%$ de la zona sur y el $28 \%$ de la zona oeste (Balsa, 2006).

Esta expansión estuvo asociada a un notable crecimiento poblacional impulsado por los inmigrantes europeos dispuestos a desarrollar la actividad en la región (Balsa, 2006). Así, se conformó una importantísima capa de

diversidad posible de "agricultores familiares", incluyendo a las "unidades unipersonales" en las que aparece el titular (o jefe del hogar) como único trabajador familiar. 
pequeños y medianos productores de hasta 500 hectáreas. Hacia los años 30, la mitad de los arrendatarios se encontraba en la franja de 250 a 500 hectáreas (Zeberio, 1993). Incluso, en la zona norte de la provincia - en la que se desarrolló la investigación en la que se basa este artículo -, era más pronunciada la tendencia a que la mayor parte de los cultivos estén en manos de pequeñas explotaciones.

Diferentes estudios fueron seńalando la emergencia de transformaciones en este segmento de productores que darían cuenta, ya a mediados del siglo $\mathrm{XX}$, de un proceso de propietarización y capitalización de las explotaciones familiares (Martínez Dougnac, 2003)

La evolución de la proporción de explotaciones agropecuarias bajo arriendo en la provincia de Buenos Aires registrada a través de información proveniente de los Censos Nacionales Agropecuarios, muestra un sostenido acceso a la propiedad desde mediados de siglo pasado. Así, mientras que en los censos de 1914, 1937 y 1947 la proporción de productores arrendatarios oscila entre el $60 \%$ y el $65 \%$ del total, para 1960 se registra una marcada caída del arriendo que explica para ese ańo sólo un $25 \%$ de la situación de los productores en la provincia de Buenos Aires en lo que se refiere a la tenencia de las tierras.

La consolidación de este sector de productores agropecuarios que acceden a la propiedad de la tierra y el inicio de un proceso de capitalización de las explotaciones da cuenta de situaciones diversas explicadas tanto por un contexto político y socio-económico favorable ${ }^{5}$ como por las estrategias desarrolladas por los propios productores. Las posibilidades de generar excedentes y ahorrar permitieron el acceso a la propiedad a través de la compra privada de tierras, así como también la existencia de programas de colonización y reparto estatal. Por su parte, el proceso de mecanización que tiene lugar en estas décadas es impulsado por la disponibilidad de créditos y la mayor facilidad para acceder a los mismos en tanto propietarios de la tierra (Martínez Dougnac, 2003).

En el plano de las estrategias más comunes utilizadas por los pequeños productores para reproducir y ampliar su explotación se reconocen las siguientes:

5 Fundamentalmente, por el congelamiento del valor de los arrendamientos pero también por la difusión de formas de financiamiento a la producción.. 
- El desarrollo de sociedades familiares en las que participaban no sólo padres e hijos sino también por parte de otros miembros de la familia extensa muy difundida para esa época. La posibilidad de disponer del trabajo de los miembros de la familia cuando se necesitaba permitió al productor incrementar su capacidad de ahorro.

- La compra de maquinaria que se difundió a partir de la década del '60 y que llevó a que algunos de estos pequeños productores puedan desarrollarse como contratistas. Como señala Urcola (2013), el proceso de modernización agraria permitió intensificar la producción de los cultivos junto con el ahorro de mano de obra, no sólo asalariada sino también familiar.

- La combinación de propiedad con arriendo o subarriendo que permitía incrementar la escala de producción y aumentaba la posibilidad de acumulación cuando había mano de obra familiar disponible.

- La diversificación productiva y económica cuando el capital acumulado lo permitía. Entre las actividades realizadas se registra la compra-venta de tierras, la consignación de ganado y la prestación de maquinarias, entre otras (Zeberio, 1993).

Sobre esta imagen de los "chacareros" convertidos en propietarios identificado como un proceso de "farmerización" que culmina en gran medida hacia comienzos de los años setenta, se instaura a principios de los años noventa un intenso debate académico para definir a este actor social. Estos trabajos parten de la discusión de los años setenta ${ }^{6}$, para analizar y conceptualizar las características de las explotaciones familiares de la región pampeana.

Tort, Bearzotti y Neiman (1991) sostienen que la "farmerización" característica de la región pampeana se asienta en una estructura productiva exclusivamente comercial y en inversiones importantes de capital (a las que no todas las explotaciones tienen acceso) que llevan a menores requerimientos laborales. "La función del trabajo aportado por el productor y su familia -aporte que aparece en principio, como resultado del tamańo y composición de la misma- ha probado ser fundamental en la explicación de la lógica y tendencias de la agricultura familiar. En primer lugar, permitiendo el control

6 Archetti y Stolen (1975), Bartolomé (1975), Vessuri (1974) 
del proceso de trabajo cuyas instancias fundamentales se organizan siguiendo las líneas de parentesco del grupo doméstico (...) Y, además, la relación familia/ trabajo se inscribe de distinta manera, dependiendo de las circunstancias, en la marcha de la actividad económica de la explotación" (Tort, Bearzotti y Neiman, 1991, p.568).

Forni y Tort (1984) también aceptan esta tendencia para una parte de los productores chacareros al afirmar que "a través de la consolidación de la propiedad y la mecanización sustitutiva de mano de obra, están dadas finalmente las condiciones para la desaparición de los chacareros y la consolidación de la "farmerización". La diferencia en la historia rural pampeana entre ambos reside básicamente en la mayor productividad de la mano de obra (vía mecanización) que, si bien siguió siendo familiar, disminuye sustancialmente en cantidad" (Forni y Tort, 1984, p.15).

A principios de los años noventa se intensifica el debate en torno a qué es lo que define de manera inequívoca el carácter familiar de una explotación agropecuaria. En la bibliografía local, autores como Murmis (1991), Mascali (1992), Cloquell (2001), Balsa (2002) y Neiman, Bardomás y Quaranta (2003) entre otros, p se basan en el porcentaje mayoritario de familiares en la composición de la mano de obra para delimitar el carácter familiar de la explotación y en la ausencia (o menor presencia) de trabajadores asalariados, así como también en el grado de autonomía que tienen para tomar decisiones productivas y de consumo.

Por su parte, Craviotti (2002) y Tort y Román (2005) enfatizan en la necesidad de que los miembros del hogar sean los responsables tanto de la gestión de la explotación como de las tareas directas de campo. Craviotti (2002) entiende por productores familiares a aquellos que "además de las tareas de gestión realizan tareas físicas en sus predios, relevantes para el funcionamiento del proceso productivo"; Tort y Román (2005) utilizan los dos criterios siguientes para diferenciar una explotación familiar de una de tipo empresarial: a) la participación directa del titular y su familia en las labores del campo, aunque se contraten trabajadores permanentes y/o eventuales; $y$ b) la responsabilidad directa del titular en la administración de la explotación, tanto en la parte comercial-financiera como en la laboral productiva, sin que exista, por lo tanto, un administrador contratado. 
Además de la centralidad del trabajo familiar como organizador de la explotación, Martínez Dougnac (2003) agrega que el objetivo de una explotación familiar se orienta esencialmente a asegurar la supervivencia de la unidad productiva, pudiendo atravesar períodos de acumulación de capital y otros de reproducción simple.

Algunos ańos más tarde se empiezan a encontrar signos de agotamiento de este marco explicativo en los nuevos escenarios en paralelo con la crisis que era evidente en ese sector. Azcuy Ameghino y Fernández (2008) utilizan el concepto de "deschacarerización" para explicar el proceso de debilitamiento o pérdida del rasgo que define a la agricultura familiar: la centralidad del aporte de trabajo familiar directo en la producción. Por su parte, Balsa (2006) también da cuenta de este proceso; lo denomina "aburguesamiento de los chacareros", y muestra que los productores familiares que lograron expandirse a través de la compra de campos y/o arriendo de predios vecinos, y que comenzaron a utilizar personal asalariado o contratistas han ido, al mismo tiempo, reduciendo drásticamente el aporte de trabajo de los distintos miembros de la familia.

\section{Las transformaciones en el modelo productivo y en la estructura agraria}

La década de los noventa significó un período de cambios para el sector de la pequeña y mediana producción pampeana. Se trató de un período durante el cual gran parte de la tradicional pequeña producción se endeudó hasta perder sus propiedades, otros se convirtieron en pequeños rentistas perdiendo su condición de productores y también hubieron transformaciones hacia empresas agropecuarias que perduraron y se adaptaron al nuevo modelo de producción. Como señalan Gras y Hernández (2010), para este período, este sector de unidades agropecuarias atravesó "procesos de descomposición y recomposición" y se introdujeron nuevos cortes en la estructura agraria a partir del desarrollo de trayectorias sociales diferentes.

En principio se destaca la caída en el número de explotaciones agropecuarias que habría afectado mayoritariamente al segmento de agricultores familiares durante la última década del siglo pasado. Se trata de 59.909 explotaciones menos para la región pampeana equivalente a un 30\% del total de unidades. 
En la provincia de Buenos Aires el descenso es de 24.415 explotaciones (32.3\%) mientras que desaparecen 4.172 explotaciones (38\%) en los partidos que pertenecen al llamado núcleo maicero ${ }^{7}$; en el caso particular del partido de Junín, se pierden 509 explotaciones, que representan el 46,7\% del total (INDEC 1988, 2002).

Esta caída en el número de explotaciones en el partido de Junín afectó especialmente al sector de explotaciones más pequeñas, ya que desaparece el $75,5 \%$ de las explotaciones de hasta 25 hectáreas y el 49,4\% entre las de 25 a 200 hectáreas. Además, en cuanto a la escala de las explotaciones, se observa que las únicas que presentan un incremento en número y participación en el total durante el período 1988-2002 son las del segmento de más de 1.000 hectáreas que se incrementan un 23,8\%, constituyen el 4,5\% de las unidades productivas del partido y controlan un $33,3 \%$ de la superficie (Cuadro 1) (Bober y Neiman, 2012).

Cuadro I - Distribución de explotaciones agropecuarias por escala de extensión 1988 - 2002. Partido de Junín.

\begin{tabular}{|c|c|c|c|c|c|c|c|c|c|c|c|c|c|c|c|c|}
\hline \multirow{2}{*}{ Año } & \multirow{2}{*}{$\begin{array}{l}\text { Total } \\
\text { EAPs }\end{array}$} & \multicolumn{3}{|c|}{ Hasta 25} & \multicolumn{3}{|c|}{ De 25 a 200} & \multicolumn{3}{|c|}{ De 200 a 500} & \multicolumn{3}{|c|}{ De 500 a 1000} & \multicolumn{3}{|c|}{ Más de 1000} \\
\hline & & $\mathbf{N}^{\circ}$ & $\%$ & $\begin{array}{l}\text { has. } \\
(\%)\end{array}$ & $\mathbf{N}^{\circ}$ & $\%$ & $\begin{array}{l}\text { has. } \\
(\%)\end{array}$ & $\mathbf{N}^{\circ}$ & $\%$ & $\begin{array}{l}\text { has. } \\
\text { (\%) }\end{array}$ & $\mathbf{N}^{\circ}$ & $\%$ & $\begin{array}{l}\text { has. } \\
(\%)\end{array}$ & $\mathrm{N}^{\circ}$ & $\%$ & $\begin{array}{l}\text { has. } \\
(\%)\end{array}$ \\
\hline 1988 & 1089 & 241 & 22,1 & 1,4 & 607 & 55.7 & 27,9 & 165 & 15,1 & 24.8 & 55 & 5,1 & 18,8 & 21 & 1,9 & 27 \\
\hline 2002 & 580 & 59 & 10,1 & 0,49 & 307 & 52.9 & 19 & 138 & 23,8 & 26.4 & 50 & 8,6 & 20,8 & 26 & 4,5 & 33,3 \\
\hline $\begin{array}{l}\% \\
88-02\end{array}$ & -46.7 & -75.5 & & & $-49,4$ & & & $-16,4$ & & & $-9,1$ & & & 23,8 & & \\
\hline
\end{tabular}

Fuente: elaboración propia en base a los Censos Nacionales Agropecuarios, INDEC, 1988 y 2002.

A partir del año 2002, cuando Argentina atraviesa una fuerte devaluación del peso y, por otro lado, se incrementan los precios internacionales de los productos agropecuarios, comienzan a presentarse nuevos cambios en el

7 Conformado por los siguientes partidos de la provincia de Buenos Aires Arrecifes, Baradero, Capitán Sarmiento, Carmen de Areco, Chacabuco, Colón, General Arenales, Junín, Pergamino, Ramallo, Rojas, Salto, San Antonio de Areco, San Nicolás y San Pedro. 
modelo productivo de la pequeña y mediana agricultura pampeana. Las principales características de estas transformaciones son la incorporación masiva de la siembra directa, el glifosato y la semilla transgénica, la creciente tendencia a la sojización de la región, la amplia difusión del contratismo de maquinaria; en este marco se observan cambios en las dinámicas familiares y en las trayectorias ocupacionales de los jóvenes de la agricultura familiar que constituyen un elemento especial de interés para comprender sus actuales condiciones de funcionamiento así como para aproximarse a una nueva definición de la misma.

El precio de las commodities en la región en estudio ha experimentado un fuerte incremento en el mercado mundial, incitado entre otras razones por la incorporación de grandes contingentes de población al mercado de consumo alimentario. Por ejemplo, "en los 14 años comprendidos entre 1994 y 2007, China pasó de comprar en los mercados internacionales 52 mil toneladas de porotos de soja a casi 31 millones de toneladas, incrementando así la cifra inicial 597 veces. Argentina acompañó esta expansión, y para 2007 cubrió aproximadamente el 30\% de las importaciones chinas totales de este producto, por detrás de Brasil y Estados Unidos" (Keller, 2008: p.1)

En el caso particular de la producción de soja, se puede observar que su precio en dólares se ha quintuplicado durante la última década (cuadro 2). Asimismo, se encuentra la misma tendencia hacia el alza de precios en el caso de otros cultivos como el maíz, el arroz y el trigo (cuadro 3).

Cuadro 2 - Evolución del precio de la soja. Cotizaciones FOB (2003 - 2012).

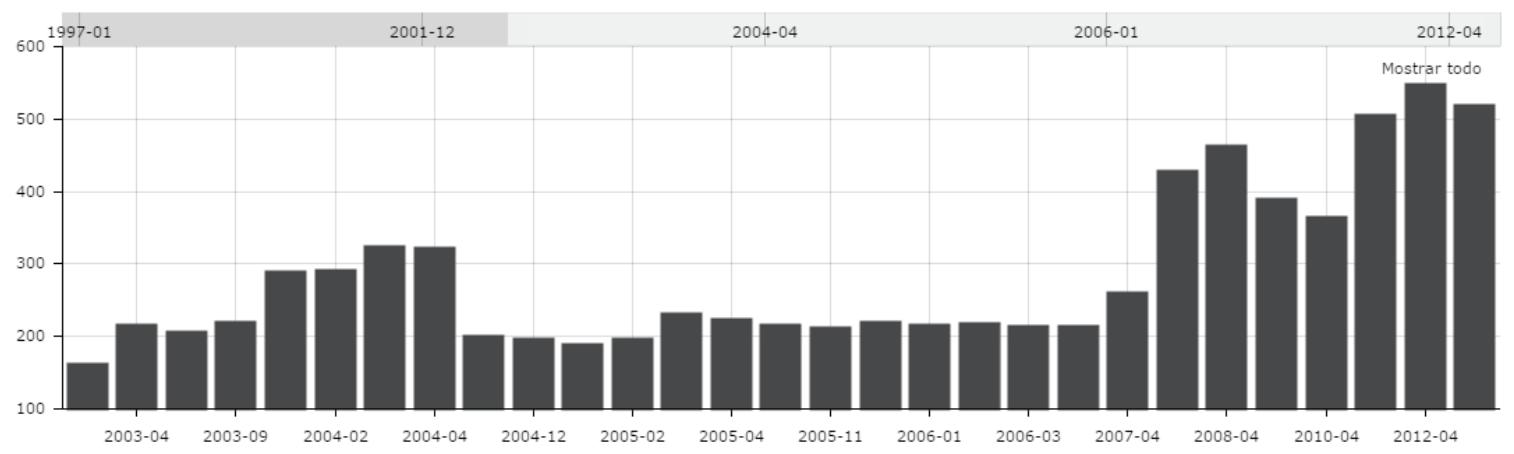

Fuente: MAGyP - Dirección de Mercados Agroalimentarios. 
Cuadro 3 - Evolución de los precios de maíz, trigo y soja, en precios constantes de 2005 (2000 - 2012)

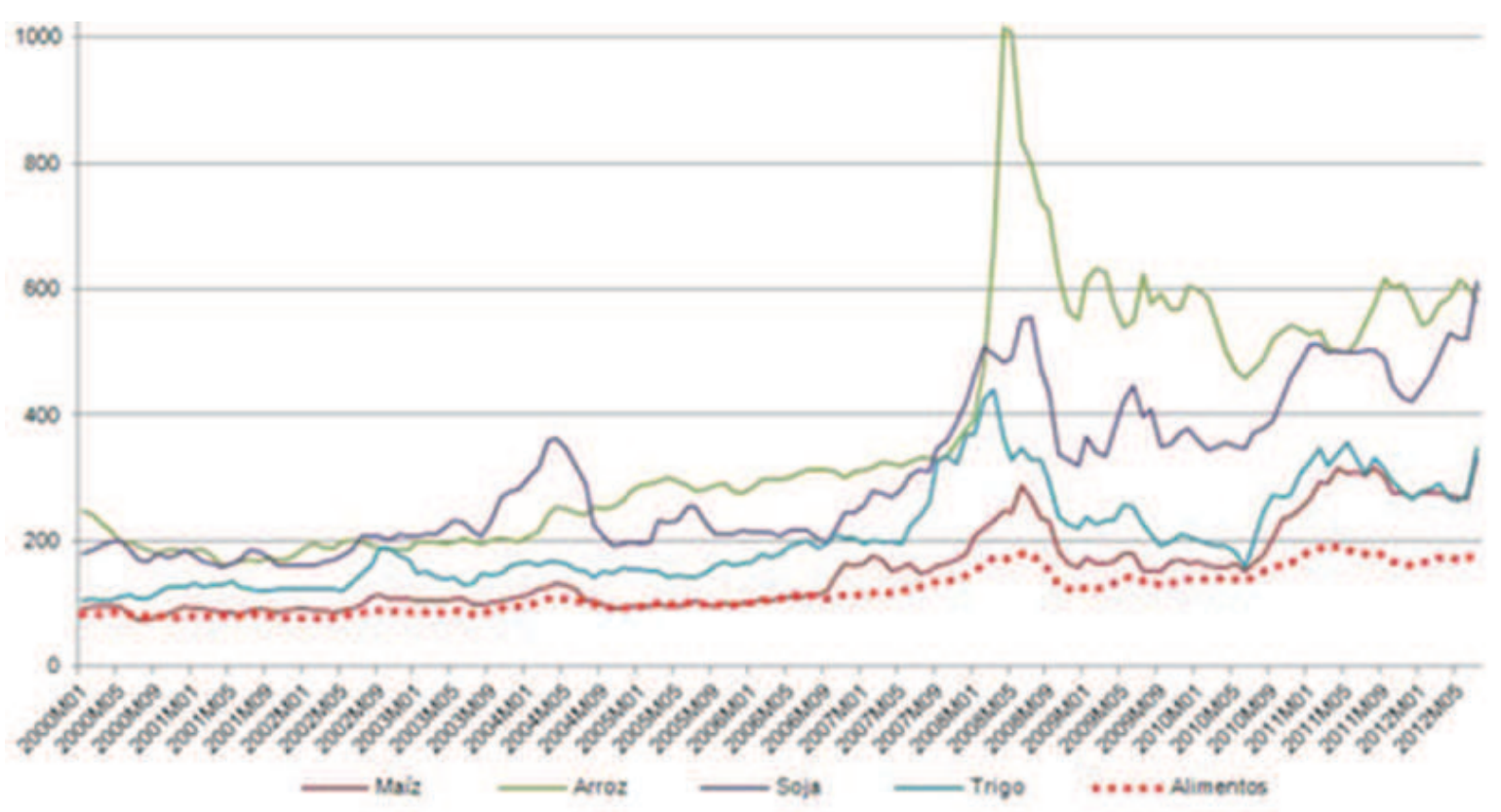

Fuente: Sistema Integrado de Información Agropecuaria,

Programa de Serviços agrícolas Provinciales, MINAGRI

La renta de la tierra, como parte constitutiva del precio de la mercancía agrícola también se incrementó en forma paralela al aumento del precio de los productos. En el cuadro 4 se puede observar el alza en el precio de la tierra, que en el caso del partido de Junín se incrementa de manera ostensible: "El valor promedio del precio de la tierra en la región aumentó alrededor del 400 por ciento en los últimos diez años y se ubica en unos 15.000 dólares por hectárea, aunque en algunos distritos trepa hasta los 20.000 dólares" (Diario La Democracia, 2011). 
Cuadro 4 - Evolución del precio de la tierra según zona productiva (1977 - 2001)

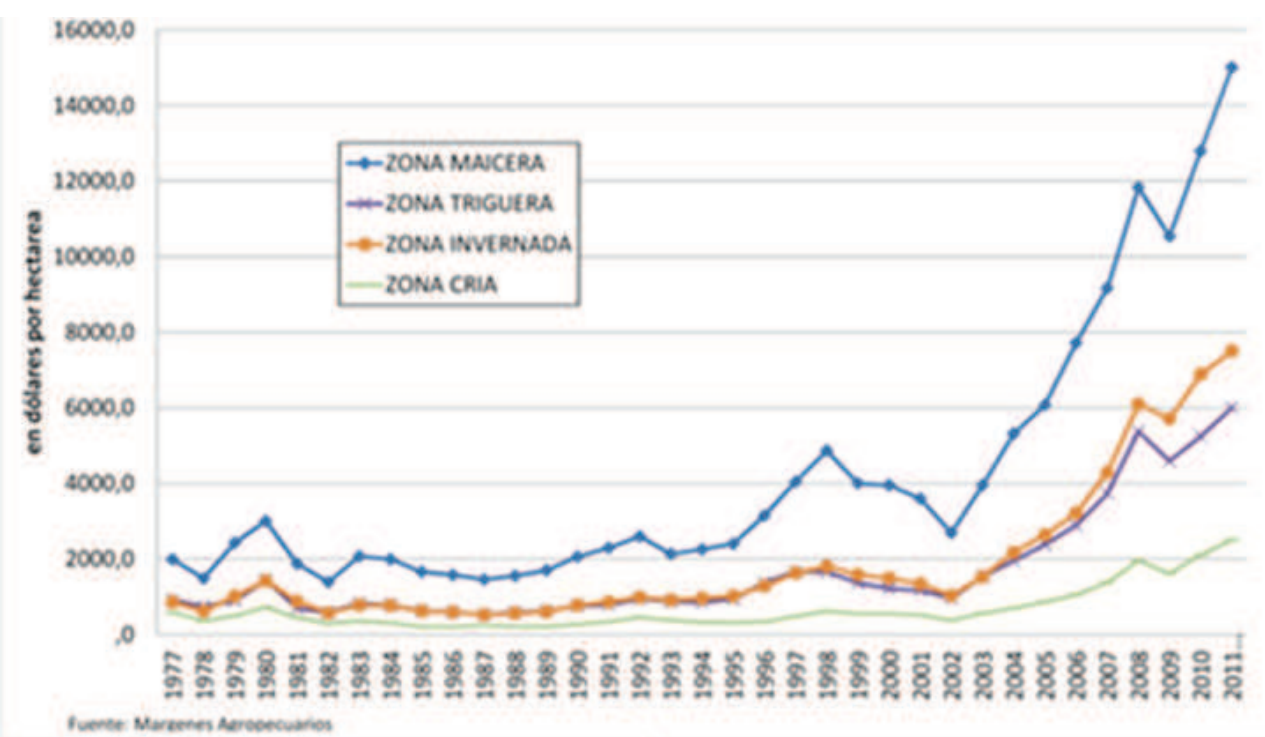

El incremento en el precio de la renta de la tierra corre paralelo a un aumento en el precio de los arrendamientos (además que se incrementan las operaciones de este tipo), que en una zona núcleo de región pampeana como el partido de Junín, alcanza a 17 quintales de soja o 450 dólares por hectárea para el año 2014 (cuadro 5).

Cuadro 5 - Evolución del precio de arrendamiento para soja en zona núcleo $(2001-2014)$

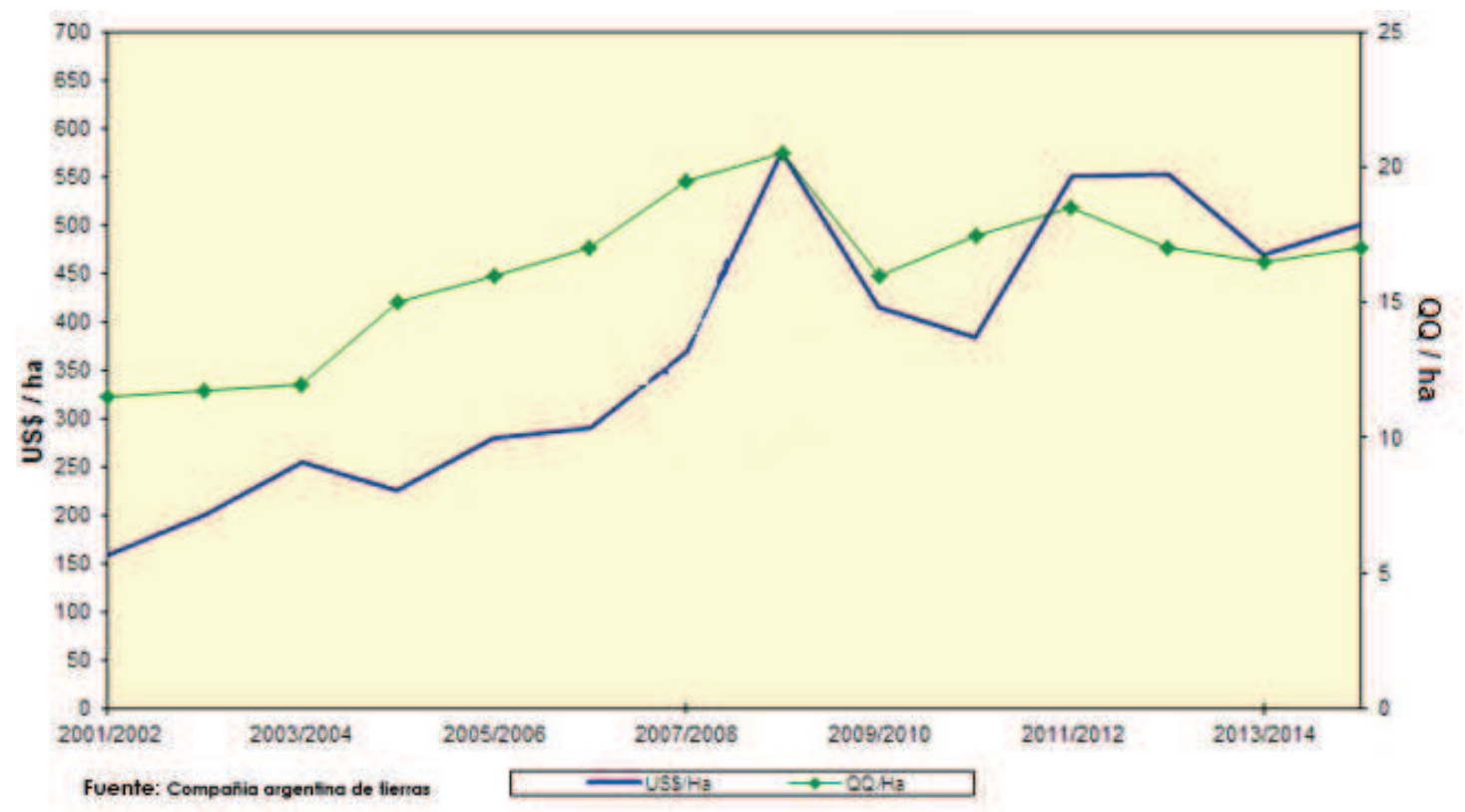


Los requerimientos de reformulación del modelo productivo de la siembra directa y la necesidad de capital para realizarlo resultaron fuertes limitaciones para las pequeñas y medianas unidades que se encontraban fuertemente endeudadas, sin acceso a nuevos créditos, sujetas a problemas en la efectivización de la sucesión - presencia de titulares de edad avanzada sin hijos a los cuales traspasar la explotación- y sometidas en algunos casos a cosechas arruinadas por factores climáticos como sequías e inundaciones. En tales circunstancias y ante los altos precios del arrendamiento, se explica la opción por el "pequeńo rentismo" de un sector importante de estas explotaciones y su desaparición como unidades productivas, incluso como estrategias para salvar la propiedad (López Castro, 2014).

El nuevo modelo productivo no sólo trae aparejados cambios en la organización de la producción por la desaparición de tareas como la roturación de los suelos, desmalezado, etc. y por la incorporación del doble cultivo trigo-soja, sino que también significa cambios en la forma en que se organiza el trabajo al interior de las unidades que pudieron reformularse y seguir produciendo.

\section{La organización del trabajo y las dinámicas familiares}

Las familias involucradas en estas unidades agropecuarias han atravesado un fuerte proceso de urbanización. Si bien se reproducen a partir de los ingresos provenientes de la agricultura, la mayoría de estas familias no residen en la explotación agropecuaria ${ }^{8}$.

Esta situación trae aparejadas transformaciones tanto en la organización del trabajo de la unidad agropecuaria como en la socialización e individuación de los diferentes miembros de la familia. Este actor social podría ser conceptualizado como perteneciente a las "capas medias" del agro argentino, no sólo por sus niveles de ingresos sino también por la adopción de algunos hábitos culturales que han caracterizado históricamente a los sectores medios urbanizados de la Argentina, en especial de la ciudad de Buenos Aires.

En cuanto a la composición y organización del trabajo al interior de estas unidades productivas, lo primero que se puede señalar es que, en el partido

8 El 30,8\% de estas familias residen en la explotación mientras que el 69,3\% vive en localidades urbanas, ya sea en la ciudad de Junin o en pequeñas localidades rurales (Neiman, 20II). 
de Junín, el 75\% de estas explotaciones combinan el trabajo del productor con el trabajo no familiar, incluyendo en esta última categoría la contratación de asalariados ya sea permanentes o transitorios y, también, a los servicios de maquinaria para la realización de tareas de siembra y/o de cosecha; sólo un 12\% de los productores cuenta, además, con la presencia de trabajadores familiares; el restante $13 \%$ está conformado por unidades en las que se desempeńa solamente el productor o el trabajo del titular aparece acompañado por el de otros integrantes del núcleo familiar (y no se registra la contratación de ninguna de las formas de trabajo no familiar arriba mencionadas) (Neiman, 2011).

Asimismo, se observa que el $61,3 \%$ de las unidades con trabajadores familiares contrata servicios de maquinaria mientras que este porcentaje alcanza al $82,3 \%$ en las explotaciones en las que no cuentan con miembros de la familia que trabajen (a excepción del productor) (Neiman, 2011); esto muestra, por un lado, la amplia difusión de la contratación de servicios y, por otro, la relación positiva entre contratación de servicios de maquinaria y la ausencia de mano de obra familiar.

La difusión de la contratación de servicios de maquinaria asociada a la menor demanda de mano de obra en el modelo de la siembra directa modificó la forma de organizar el trabajo y la producción y el contratista de maquinarias se instauró como un actor clave en la expansión del modelo y en la estandarización de las formas de producción, incluso para la "agricultura familiar".

En lo referido a los cambios ligados a la adopción de hábitos característicos de las clases medias de tipo urbano, es necesario observar las relaciones que mantienen los miembros de las familias con su entorno. Las características de la socialización y de los procesos de individuación ${ }^{9}$ de los miembros de la familia, particularmente de los/as hijos/as de los productores, se pueden resumir en el acceso a la educación formal no sólo secundaria si no también universitaria, la posibilidad de ocupación en empleos tanto profesionales

9 Esquemáticamente, se puede distinguir la socialización de la individuación ya que la primera "estudia el proceso de fabricación socio-psicológico del individuo (...), el problema de la constitución del sujeto [mientras que] la individuación se interesa, desde una perspectiva socio-histórica, por el tipo de individuo que es estructuralmente fabricado en una sociedad" (Araujo y Martuccelli, 20 I0: 79). Esta perspectiva apunta al estudio de la creciente singularización de las trayectorias personales y la importancia de su análisis para la comprensión del desenvolvimiento de los individuos en los contextos socio-históricos actuales. 
como en el comercio local y también ciertos cambios en la conformación de las familias.

Las tendencias encontradas al interior de los grupos familiares son: el número reducido de miembros por familia ${ }^{10}$ (que implica planificación de familias chicas); la convivencia de hasta dos generaciones expresado en el muy bajo porcentaje de familias extensas o compuestas ${ }^{11}$; la formación más tardía de los matrimonios de la generación de los hijos (prolongación del período de soltería) y el reducido número de hijos por familia (tanto por planificación como por la mencionada formación tardía de los matrimonios).

Hervieu (1996) sostiene que la concepción del rol de jefe de familia se ha modificado y que los agricultores modernos jóvenes se casan cada vez más con mujeres "no agrícolas" y con empleos urbanos. A esta situación, que contrasta con la imagen tradicional del agricultor "dueño" de su familia y su explotación, habría que añadirle un segundo fenómeno que refuerza la tendencia anterior, referido a los efectos de persistencia e incluso del aumento de las situaciones de soltería entre los agricultores jóvenes. La totalidad de los hijos que viven con sus padres y no trabajan en la unidad son solteros, porcentaje que se reduce al $68,2 \%$ si se considera a los hijos que trabajan en la explotación agropecuaria (vivan o no en el hogar familiar) (Neiman, 2011).

En cuanto a los efectos de estos cambios en la organización de la unidad agropecuaria, se observa un proceso de desvinculación familiar de la unidad productiva debido a que, por un lado, el desarrollo del contratismo de maquinaria llevó a que estas unidades puedan sostenerse con el trabajo del productor y sin la presencia de ningún otro trabajador familiar y, por el otro, a que por los procesos de individuación que atraviesan los hijos, buscan desarrollar sus propias trayectorias con mayores márgenes de autonomía.

Cuando se encuentran miembros de la familia que no se desempeñan en la explotación y lo hacen exclusivamente en otras ocupaciones, mayoritariamente no agrícolas, se conforma un segmento particular de "hogares pluriactivos". Estas inserciones laborales pueden expresar una estrategia de diversificación

10 La cantidad promedio de miembros por familia agrícola de Junín es de 3,03 personas (Neiman, 201 I), apenas superior a la de la ciudad de Buenos Aires donde es de 2,7 miembros (INDEC, 200I).

II Las familias extensas y compuestas representan sólo el I1\%, los hogares unipersonales, 15\% y los hogares con núcleo familiar completo o incompleto alcanzan al 74\% (Neiman, 2011). 
de la base económica de las unidades familiares, de apuntalamiento de la economía vía la integración de ingresos "externos" a la economía familiar o, incluso, de una decisión de mantener el vínculo de los miembros del hogar con el núcleo original y eventualmente funcionar como reemplazo generacional.

El hecho que familiares que no se desempeñan en la explotación agropecuaria, como puede ser la esposa o algún/a hijo/a del productor, se desarrollen laboralmente fuera de la unidad o incluso del sector agropecuario no significa que la organización laboral y productiva de la explotación se vea modificada. Bajo esta condición se han podido identificar varias situaciones. Por un lado, están los/as hijos/as que mantienen su residencia en el hogar de origen, que tienen un diploma universitario o terciario y cuya inserción laboral puede ser el inicio de una trayectoria profesional personal aunque mientras tanto realicen aportes al núcleo familiar pero no constituyen mano de obra de la unidad productiva.

Otro caso particular pero que tiene alguna difusión lo constituye la inserción de un integrante de la familia en algún emprendimiento productivo generado a partir de una inversión - generalmente de escala reducida - de fondos provenientes de la misma actividad productiva agrícola.

Asimismo, una tercera situación identificada en la investigación de campo le corresponde a las cónyuges de los titulares que se insertan en actividades comerciales o como docentes en establecimientos educativos de la zona; en general, se trata de inserciones a tiempo parcial ya que parte de su jornada la dedican a la actividad doméstica o, incluso, a alguna tarea de gestión que requiera la economía agraria de la finca (Neiman, Berger y Neiman, 2013).

Cuando los/s hijos/as de los productores comienzan a desarrollar sus propias trayectorias laborales también se ven afectados los procesos hereditarios. "Salir" de la unidad productiva para desempeñarse laboralmente no significa sin embargo una renuncia de su participación en el reparto de la herencia de la propiedad. En muchas ocasiones, los/as hijos/as se insertan laboralmente en el mercado después de haber completado una carrera universitaria y esto pone en cuestión el concepto de "hereda la tierra quien la trabaja" (Neiman, 2013) y da lugar al surgimiento de futuros "herederos no rurales".

Se distinguen dos etapas principales del proceso sucesorio que presentan, a su vez, diferentes tipos de arreglos intrafamiliares. Por un lado, se encuentra 
una etapa en que el productor comienza a compartir espacios de gestión de la explotación con su descendencia o parte de ella. Al principio de esta etapa, uno de los arreglos informales posibles es el otorgamiento de una remuneración a los hijos (o al único hijo) que se incorporan al trabajo de la unidad. Transcurrido un tiempo, se desarrollan otros tipos de arreglos tendientes a compartir los espacios de decisión de la empresa como la formación de sociedades entre padres e hijo/s (S.R.L, S.A. o Sociedades de hecho). También se registran casos en que uno de los hijos se fue incorporando al trabajo de la unidad sin recibir un pago, hasta la conformación de la sociedad. Asimismo, existen unidades en que ningún hijo estaba dispuesto a quedarse trabajando en la unidad, truncando este proceso. En estos casos, el padre productor suele convertirse en un propietario rentista, abandonando su condición de productor.

Por otro lado, en una segunda etapa, se debe resolver cómo se produce la transferencia legal de la propiedad de la tierra. En estos casos, se observa más apego a la ley para la distribución de la herencia entre los/as hijos/as. Toda sucesión hereditaria comprende, dentro de las estipulaciones legales, dos fracciones: la legítima, que corresponde a los herederos forzosos y la disponible -actualmente una quinta parte- sobre la cual el testador puede manifestar su voluntad de destino, respetando las estipulaciones del Código Civil (artículo 3280). Una vez distribuida la propiedad de la tierra entre la descendencia, es común el arrendamiento intrafamiliar para la gestión de la explotación agropecuaria.

Cuando se analizan las diferentes estrategias desplegadas por las familias para la reproducción de la unidad se distinguen ciertas rupturas con respecto a la imagen tradicional de la "agricultura familiar" o de los "chacareros" de las zonas núcleo de la región pampeana:

- En el modelo productivo actual, la fuerza de trabajo familiar no se presenta como un recurso que otorgue flexibilidad a la organización de la producción; las familias son de tipo nuclear y la formación de sociedades apunta a resolver la gestión de la explotación y el relevo generacional.

- La generalización de la contratación de servicios de maquinaria llevó a que las unidades no necesiten contar con la tecnología para intensificar la producción. Incluso estas unidades deciden ofrecer servicios 
cuando un hijo del productor busca desarrollar una actividad de forma autónoma.

- La decisión de arrendar tierras y aumentar la escala de producción no se encuentra vinculada directamente con la disponibilidad de mano de obra, si no que se trata de una decisión de tipo "empresarial" de costo-oportunidad.

- El desarrollo de la pluriactividad aparece ligado a los proyectos personales de los miembros de las familias más que a una estrategia de diversificación productiva.

- En los procesos sucesorios, lo que está en juego es la transmisión de una empresa y de la propiedad de la tierra y no un "oficio de agricultor".

\section{Conclusiones}

En el contexto post-devaluación que se inicia a comienzos de la década del 2000 en la Argentina, los pequeños y medianos productores de la región pampeana que pudieron conservar sus tierras, tuvieron dos destinos posibles. Por un lado, se encuentran aquéllos que, en tanto propietarios de la tierra, decidieron arrendarlas y establecer su residencia definitiva en un centro urbano - en este caso la ciudad de Junín- perdiendo su condición de productor. Por el otro, los que permanecieron en la actividad lo hicieron con algunos cambios en la organización del trabajo y de la familia.

En este escenario, el trabajo dejó de ser el argumento articulador de la definición de la agricultura tradicionalmente denominada "familiar". Esto se debe, por un lado, a las características de una actividad cada vez menos demandante de mano de obra y, por el otro, a los cambios en las dinámicas familiares y al desenvolvimiento de trayectorias individualizadas por parte de la generación de los/as hijos/as de los productores. Asimismo, la creciente "managerización" de las unidades agropecuarias influyó en esta dirección. Al desarrollarse la gestión de la empresa, se recurrió al uso de los saberes profesionales tanto en la esfera productiva a través la contratación de servicios técnicos como en la económico-financiera y legal, mediante el vínculo con contadores y abogados para llevar a cabo la dirección de la empresa agropecuaria de la manera más profesionalizada posible e incluso para intervenir en la 
conformación de sociedades familiares y en el proceso sucesorio.

El productor y su familia, en búsqueda de mejores condiciones de vivienda y acceso a servicios, pasaron a residir en la ciudad, contratar todos los servicios que la producción demande, y mediante el traslado diario del productor al campo -y, eventualmente, de los hijos del productor-, llevar a cabo la actividad productiva. El desarrollo de los espacios de gestión de la empresa agropecuaria, su profesionalización y la dedicación de los trabajadores familiares en estas tareas derriba la especificidad agrícola del trabajo agropecuario, así como también tiene impacto en las relaciones entre géneros y generaciones.

La generación de los productores logra permanecer hasta una edad avanzada en la dirección de la empresa familiar debido a que no necesita desarrollar las actividades físicas que demanda el trabajo agropecuario. La generación de los hijos busca desarrollar autónomamente sus trayectorias laborales sin cerrar la posibilidad de regresar en un futuro a la gestión de la empresa agropecuaria (especialmente, los hijos varones). Las hijas mujeres también atraviesan estos procesos de individuación cuando van tomando sus propias decisiones laborales, aunque esto no significa una renuncia a los derechos sobre el patrimonio familiar en el momento del relevo generacional.

Este tipo de relación entre generaciones que garantiza la sucesión de la unidad y el relevo del titular se produce en un contexto económicoproductivo favorable y de alta rentabilidad. La decisión de los hijos de reingresar para gestionar la empresa agropecuaria podría estar condicionada por la permanencia del contexto económico beneficioso para la actividad, especialmente en comparación con sus posibles inserciones profesionales individuales.

En la discusión teórica de comienzos del 2000 se empezaba a vislumbrar el conflicto que generaba la definición de este actor social en base al origen familiar de sus trabajadores. Así, se consideró la preservación familiar del trabajo de gestión y de la toma de decisiones como el elemento que todavía podía revitalizar el concepto de "agricultura familiar" (Craviotti, 2002, Tort y Román, 2005). Pocos años después, Balsa (2006) y Azcuy Ameghino y Fernández (2008) también comenzaron a dar cuenta de lo que significaba, en términos teóricos y políticos, la pérdida de la centralidad del aporte del trabajo familiar en estas unidades. 
Los procesos de cambios productivos, económicos pero también familiares y culturales llevaron a que "el origen y la composición de la mano de obra" deje de funcionar como elemento aglutinador y distintivo de las unidades familiares de la zona núcleo de la región pampeana. Sin embargo, estos mismos procesos son los que llevaron a la combinación de arreglos económicos y no económicos (que se corresponden con criterios afectivos y familiares) para mantener la empresa familiar en funcionamiento pero también para responder a los proyectos de vida de los miembros de la familia. Entre estos arreglos se reconocen la remuneración al trabajo familiar, la formación de sociedades familiares, el arrendamiento de tierras a parientes, la manutención de los/as hijos/as durante su formación profesional, el incentivo al desarrollo de trayectorias profesionales individuales a través de educación, la facilitación para el desempeño de actividades autónomas (por ejemplo, la prestación de maquinaria de la unidad agropecuaria), entre otros.

La forma en que se reconfigura la relación entre unidad productiva y familia presenta nuevos rasgos cuando el aporte de trabajo familiar deja de articular ambos espacios. No obstante, el despliegue de una batería de arreglos de distinto tipo muestra que se trata de un actor social particular que se sigue diferenciando de otros actores de la estructura agraria pampeana ya que necesita responder a demandas de índole económica y no económica de los diferentes miembros de la familia para persistir como unidad.

\section{Bibliografía}

ARAUJO, K.; MARTUCCELLI, D. "La individuación y el trabajo de los individuos" en Educação e Pesquisa, v. 36, n. especial, São Paulo, 2010.

ARCHETTI, E.; STÖLEN, K. Explotación familiar y acumulación de capital en el campo argentino. Buenos Aires: Siglo XXI, 1975.

AZCUY AMEGHINO, E.; FERNÁNDEZ, D. “Causas, mecanismos, problemas y debates en torno al proceso de concentración del capital agrario en la región pampeana: 1988-2007” en V Jornadas de Investigación y Debate Trabajo, Propiedad y Tecnología en la Argentina rural del Siglo XX, Universidad Nacional de Quilmes, Bernalv 2008.

BALSA, J. La crisis del 30 en el agro pampeano. Buenos Aires: Centro Editor de América Latina, 1994. 
BALSA, J. “Expansión agrícola y transformaciones sociales en el agro pampeano, 1969-1988”. Revista interdisciplinaria de estudios agrarios $\mathrm{N}^{\circ} 16$. Buenos Aires: CIEA, 2002.

BALSA, J. El desvanecimiento del mundo chacarero. Transformaciones sociales en la agricultura bonaerense 1937-1988. Buenos Aires: Universidad Nacional de Quilmes Editorial, 2006.

BARSKY, O.; PUCCIARELLI, A. "Cambios en el tamaño y régimen de tenencia de las explotaciones agrarias pampeanas". Desarrollo agrario pampeano. Buenos Aires: INDECINTA-IICA, 1991

BARTOLOMÉ, L. "Colonos, plantadores y agroindustria. La explotación agrícola familiar en el sudeste de Misiones". Desarrollo económico, v. 15, n. 58, Buenos Aires, 1975.

BOBER, G.; NEIMAN, M. "La agricultura capitalista entre el campo y la ciudad. Estudio de dos casos en la región pampeana argentina”. Revista eutopia, n. 3. Quito: FLACSO, 2012.

CLOQUELL, S. "Transformaciones en el área agrícola del sur de Santa Fe: las estrategias de los productores familiares capitalizados". Revista de investigaciones de la Facultad de Ciencias Agrarias - UNR, Año 1, n. 1, Buenos Aires, 2001.

CRAVIOTTI, C. "Configuraciones socio-productivas y tipos de pluriactividad: los productores familiares de Junín y Mercedes". Revista interdisciplinaria de estudios agrarios, n. 7, v. 17, Buenos Aires: CIEA, 2002.

Diario La Democracia "Los campos de la zona valen cada vez más" en http://www. diariodemocracia.com/notas/2011/5/25/locales-25496.asp , 2014.

FORNI, F.; TORT, M. I. Las explotaciones familiares en la producción de cereales de la región pampeana argentina, Buenos Aires: CEIL, 1984.

GRAS, C.; HERNANDEZ, V. La argentina rural. De la agricultura familiar a los agronegocios. Buenos Aires: Editorial Biblos, 2009.

HERVIEU, B. "La década de todas las rupturas" en Los campos del futuro. Madrid: Serie Estudios, 1996.

INDEC Censo Nacional Agropecuario 1988 y 2002, Censo Nacional de Población 2001, Buenos Aires.

KELLER, M. "Argentina, China, y las oleaginosas". Revista alimentos argentinos, n. 43. Buenos Aires, 2008.

LÓPEZ CASTRO, N. "De chacareros a rentistas: trayectorias de abandono de la actividad agropecuaria en el SO bonaerense" (Puán y Adolfo Alsina, 1988-2012). Revista mundo agrario, v. 15 p. 1-39, La Plata, 2014. 
MARTÍNEZ DOUGNAC, G. "La agricultura familiar en la región pampeana. Hacia un intento de definición del sujeto social”. III Jornadas Internacionales de Historia Económica. Montevideo: Asociación Uruguaya de Historia Económica, 2003.

MASCALI, H. "Mercado de alquiler de tierras y ciclo doméstico en explotaciones familiares". Explotaciones familiares del agro pampeano, Tomo 1. Buenos Aires: Centro Editor de América Latina, 1992.

MURMIS, M. "Temas en la sociología rural latinoamericana: restructuración, desestructuración y problemas de excluidos e incluidos”. Ruralia, n. 5, Buenos Aires, 1994.

NEIMAN, G., BERGER, M.; NEIMAN, M. "La pluriactividad entre pequeńos y medianos productores de la provincia de Buenos Aires. Contextos productivos, familia y trabajo". Adaptaciones y transformaciones en las agriculturas pampeanaa inicios de siglo XXI (Comps. Gasselin, P., Cloquell, S; Mosciaro, M.). Buenos Aires: Ediciones Ciccus, 2013.

NEIMAN, G., BARDOMÁS, S.; QUARANTA, G. "El trabajo en el agro pampeano. Análisis de la demanda de trabajadores asalariados." Revista Interdisciplinaria de Estudios Agrarios No 19. Buenos Aires: CIEA, 2003.

NEIMAN, M. La agricultura familiar en la región pampeana. Nuevos vínculos familiares y transformaciones en las unidades de producción, Tesis de Doctorado (Doctorado en Ciencias Sociales). Facultad de Ciencias Sociales, Universidad de Buenos Aires, 2011.

NEMIROWSKY, L. Estructura económica y orientación política de la agricultura en la República Argentina. Buenos Aires: Menéndez, 1933.

ODDONE, J. La burguesía terrateniente argentina. Buenos Aires: Ediciones populares argentinas, 1956.

SARTELLI, E. "La vida secreta de las plantas: el proletariado agrícola pampeano y su participación en la producción rural”. XI Jornadas de Historia Económica. Córdoba, 1994.

SCOBIE, J. Revolución en las pampas. Historia social del trigo argentino 1860-1910. Buenos Aires: Solar/Hachette, 1968.

Sistema Integral de información Agropecuaria (SIIA). Programa de Servicios Agrícolas Provinciales, Ministerio de Agricultura, Ganadería y Pesca de la Nación. www.siia.gov.ar.

TENEMBAUM, J. Orientación económica de la agricultura argentina. Buenos Aires: Losada, 1946.

TORT, M.I. y ROMÁN, M. "Explotaciones familiares: diversidad de conceptos y criterios operativos". Productores familiares pampeanos: hacia la comprensión de similitudes y 
diferencias zonales. María del Carmen González comp. Buenos Aires: Editorial Astralib, 2005.

TORT, M.I., BEARZOTI, S.; NEIMAN, G. "Trabajo y producción en las explotaciones familiares". El desarrollo agropecuario pampeano. O. Barsky comp. Buenos Aires: GEL Editores, 1991.

URCOLA, M. "Estrategias socio-productivas y agricultura familiar: las nuevas tecnologías y sus implicancias en las relaciones familiares y productivas en una localidad del sur santafesino". Mundo agrario, v. 13, n. 26. La Plata, 2013.

VESSURI, H. "La explotación agrícola familiar en el contexto de un sistema de plantación: un caso de la provincia de Tucumán”. Desarrollo económico, v. 15, n. 58, Buenos Aires, 1974.

ZEBERIO, B. "La situación de los chacareros arrendatarios en la pampa húmeda. Una discusión inacabada”. Huellas en la tierra. Indios, agricultores y hacendados en la pampa bonaerense. Mandrini, R. y Reguera, A. (comps.). Tandil: IEHS, 1993.

\section{O devir recente da agricultura familiar no capitalismo agrário na região dos pampas argentinos}

\section{Resumo}

O desenvolvimento do capitalismo agrário na região pampeana envolveu, desde cedo, a pequenas e medianas explorações agropecuárias de origem familiar, imersas na produção destinada aos mercados internacionais. No entanto, apresentavam características que as distinguiam dos grandes produtores da região, principalmente, pela origem familiar de sua mão de obra. No seu devir histórico, estas unidades se viram transformadas tanto sua organização laboral-produtiva, bem como suas dinâmicas familiares. Neste artigo se mostram estas mudanças e se reflete sobre a dificuldade de definir este ator social em função das antigas particularidades, buscando entender as características que o mantem distinto. A abordagem metodológica é qualitativa e se baseia na análise de entrevistas em profundidade realizadas com produtores, seus filhos, filhas e esposas e com profissionais no distrito de Junín, província de Buenos Aires. Também se recorre a fontes secundárias para explicar os fatores relacionados com a expansão produtiva e com o preço da terra.

Palavras-chave: produção familiar, região pampeana, agricultura, famílias. 


\section{The recent development of the family farm in the agrarian capitalism of the Argentinean Pampas}

\section{Abstract}

The development of agrarian capitalism in the so called Pampas region of Argentina early involved small and medium-size family farms, immersed in commodities' production for international markets. These farms had characteristics that distinguished them from the large producers in the region, mainly because the family origin of their labor. In its historical evolution, these units have transformed both their labor-productive organization and their family dynamics. This article shows these changes and discusses the difficulty for defining this social actor in terms of its past characteristics, at the same time that it seeks to understand its current distinguishing features. The methodological approach is qualitative and is based on the analysis of in-depth interviews with farmers, their sons/daughters and wives, and professional staff linked to those farms. In addition, secondary sources of information are used to explain the structural factors related to the expansion of production and the rise of the land prices. The research was carried in the area of Junín, at the northwest region of the Province of Buenos Aires.

Keywords: Family farm. Pampas. Agriculture. Families.

Recebido em 10/12/2015

Aceito em 10/10/2016 